\title{
Study of the Convergence in State Estimators for LTI Systems with Event Detection
}

\author{
Juan C. Posada, ${ }^{1}$ Manuel J. Betancur, ${ }^{2}$ Jaime A. Moreno, ${ }^{3}$ \\ Rubén D. Guerra, ${ }^{1}$ and Martin A. Tamayo ${ }^{4}$ \\ ${ }^{1}$ Instituto Tecnológico Metropolitano (ITM), Medellín, Colombia \\ ${ }^{2}$ Facultad IEE, Universidad Pontificia Bolivariana, Circular 1 No. 70-01, Medellín, Colombia \\ ${ }^{3}$ Automation Department, Engineering Institute, UNAM, 4500 Mexico City, Mexico \\ ${ }^{4}$ EAFIT University, Medellin, Colombia
}

Correspondence should be addressed to Juan C. Posada; juancposada@itm.edu.co

Received 4 November 2015; Accepted 17 February 2016

Academic Editor: Hung-Yuan Chung

Copyright (C) 2016 Juan C. Posada et al. This is an open access article distributed under the Creative Commons Attribution License, which permits unrestricted use, distribution, and reproduction in any medium, provided the original work is properly cited.

\begin{abstract}
The methods frequently used to estimate the state of an LTI system require that the precise value of the output variable is known at all times, or at equidistant sampling times. In LTI systems, in which the output signal is measured through binary sensors (detectors), the traditional way of state observers design is not applicable even though the system has a complete observability matrix. This type of state observers design is known as passive. It is necessary, then, to introduce a new state estimation technique, which allows reckoning the state from the information of the variable's crossing through a detector's action threshold (switch). This paper seeks, therefore, to study the convergence in this type of estimators in finite time, allowing establishing, theoretically, whether some family of the proposed models can be estimated in a convergent way through the use of the estimation technique based on events.
\end{abstract}

\section{Introduction}

In LTI system, where the output signal is measured through binary sensors (detectors), the traditional design form of state observers is not applicable, even though the system has a complete observability matrix. This type of state observer design is known as passive. Detectors are used in an ample system gamut like photoelectrical detectors, to detect the presence; oxygen detectors, to control automobile emissions; 1-bit quantizers, for analogue to digital conversion; and optical encoders, for position detection, among others. The big difficulty offered by the systems where this type of detectors are used resides in the fact that the information is very limited for modeling, identifying, estimating, controlling, and detecting failures [1].

The addressed issue involves LTI systems where the input variable is supposed to be known, but the output is available through a detector that sends a signal when the variable reaches some given value. Even though the basic idea can be taken to the case where there are multiple detectors, with different thresholds, this work will focus on the single detector case.

The detector's available information accounts only for the crossing of the variable by the thresholds of the detector, without knowing the crossing direction. The instant of time of the crossing can be then known with certainty. This type of nonperiodical sampling is known as Lebesgue sampling [2]. The Lebesgue sampling, or events based sampling, is an alternative for the Riemann sampling. The systems with Lebesgue sampling are more difficult to analyze than the Riemann sampling system [3]. Nevertheless, by its conceptual simplicity, the Lebesgue sampling has been recently used in feedback systems.

The simulation of hybrid systems is needed due to the nature of the studied system, where continuous and discrete dynamics are present; this type of simulations can be developed using different tools like Matlab/Simulink and Ecosim. 
In [4], the estimation technique for an LTI system was introduced:

$$
\sum L:\left\{\begin{array}{l}
\dot{x}(t)=A x(t)+B u(t), \quad x(0)=x_{0} \\
y(t)=C x(t)
\end{array}\right.
$$

where $x \in \mathbb{R}^{n}$ is the state variable, $u \in \mathbb{R}^{m}$ is the input variable, and $y \in \mathbb{R}$ is the output scalar of the system, and $A, B$, and $C$ are real matrix of proper dimensions. The objective is to estimate the value of $x(t)$ at all times $t \in \mathbb{R}^{+}$ given that the input $u(t)$ is known at all times but the only information regarding the output $y(t)$ is given by a binary sensor, indicating that $y$ has reached some constant threshold value $Y \in \mathbb{R}$. This means, the instants of time $t_{i}, i=1,2, \ldots$, in which the output has reached the threshold, are known; that is, $y\left(t_{i}\right)=Y$. In their work, Moreno et al. gave an observability (passive) characterization for this Lebesgue sampling case.

The possibility of reconstructing the initial state of (1) depends not only on an intrinsic characteristic of the system, but also on the obtained sampling. In system (1), together with a sampling sequence, $S=\left\{t_{1}, t_{2}, \ldots, t_{N}\right\}$, where $N$, the number of samplings $N \in \mathbb{Z} \cup\{0\}$, is said to be Lebesgue sampling observable if it is possible to calculate, in a unique way, the initial state $x_{0}$, from the sampling sequences $Y$ and $u(t)$. If, after certain sampling interval, the observability is obtained, it is possible to reconstruct the state of the system for all $t$.

Assuming that the observation process began in $t_{0}$, so a Lebesgue sequence of sampling exists as $S=\left\{t_{1}, \geq t_{0}, i=\right.$ $1,2, \ldots\}$, with $t_{i}<t_{i}+1$, in which $y\left(t_{i}\right)=Y$; the measurement process can be presented like impulsive actions $y\left(t_{i}\right) \delta\left(t-t_{i}\right)$ that happen at detection times $t_{i}$ where $\delta(t)$ is the Dirac function. The measurement can also be expressed like

$$
y(t)=\sum_{i=1}^{\infty} y\left(t_{i}\right) \delta\left(t-t_{i}\right)
$$

Proposition 1. The actual state $x(t)$ of (1) satisfies

$$
P(t) x(t)=R(t)
$$

where

$$
\begin{aligned}
P(t) & \triangleq \int_{t_{0}}^{t} e^{A^{T^{(\tau-t)}}} C^{T} C e^{A(\tau-t)} \delta\left(\tau-t_{i}\right) d \tau \\
R(t) & \triangleq \int_{t_{0}}^{t} e^{A^{T(\tau-t)}} C^{T}\left[y(\tau)-C \int_{t}^{\tau} e^{A^{(\tau-t)}} B u(\sigma) d \sigma\right] \\
\cdot & \delta\left(\tau-t_{i}\right) d \tau
\end{aligned}
$$

can be calculated from measured data and the system model; if $\sum L$ is Lebesgue sampling observable with measurement $S_{t}$ sequence, generated during the observation interval $\left[t_{0}, t\right]$, then $x(t)$ can be determined uniquely from (3).

In [4] (3) was applied to an illustrative example using simulation. Some authors have investigated state estimators with event detection for another application as [5-7].
However, a formal support to determine the estimation convergence, in finite time, was not given. In our work we address this issue. Under certain conditions, this will permit establishing, in theory, if some family of proposed models can be estimated in a convergent way by using the event based estimation technique.

\section{Preliminaries}

For the paper terminology comprehension, it is necessary to understand, generically, what conforms the distribution theory and, also, to revise some definitions.

2.1. Distribution Theory. The distribution theory generalizes the concept of "function," work that was initiated by the British physician Paul Dirac, whom, close to the end of the twenties, introduced a very important concept known as the "delta function," in connection with his studies about quantum mechanics, which has the property of being equal to zero at all times, excluding the origin where its behavior is so that the defined integral in all $\mathbb{R}^{n}$ is one. The mentioned delta is not considered a function in the ordinary sense but a distribution. Starting from here and with the objective of giving a mathematical stringency, a coherent and complete recognized theory was originated as the distributions theory, created by a French scientist, Schwartz. Later the Soviet scientist, Sobolev, released his generalized functions theory, which is an extension of the distributions theory, introducing operator conditions to the function over a space of functions [8].

For the convergence study at hand, the distribution, or generalized functions, will be used; they behave as a mathematics object by generalizing the function notion, extending the concept of differentiation to all the local integrable functions.

According to [9], the distribution theory extends the differential calculus to certain lineal and continuous forms, defined in a topologic space of infinitely differentiable functions with compact support; these lineal and continuous forms are called distributions or generalized functions. This type of functions has greater coverage than the differentiable functions type in an ordinary sense.

Proposition 2. Let $\Omega$ be an open set of $\mathbb{R}^{n}$ :

$$
\begin{aligned}
& D(\Omega)=C_{0}^{\infty}(\Omega)=\{\varphi \\
& \left.\quad \in C^{\infty}(\Omega): \operatorname{sop} \varphi \text { is a compact contained in } \Omega\right\},
\end{aligned}
$$

where $\operatorname{sop} \varphi=\{\overline{x \in \Omega: \varphi(x) \neq 0}\} . D(\Omega)$ denotes the vector space of the sampling functions. If $K$ is a compact of $\Omega$, then $D_{K}(\Omega)=\left\{\varphi \in C^{\infty}(\Omega): \operatorname{sop} \varphi \subset K\right\}$.

One typical function of $\mathscr{D}(\Omega)$ is

$$
\varphi(x)= \begin{cases}0 & \text { if }|x| \geq 1 \\ C \exp \left(\frac{1}{|x|^{2-1}}\right) & \text { if }|x|<1\end{cases}
$$


where $x=\left(x_{1}, \ldots, x_{n}\right) \in \mathbb{R}^{n}$ and $|x|=\sqrt{x_{1}^{2}+\cdots+x_{n}^{2}}$, and the constant $C$ is chosen in a way that $\int_{\mathbb{R}^{n}} \varphi(x) d x=1 ; \varphi \in$ $C^{\infty}\left(\mathbb{R}^{n}\right)$ and it supports the unitary ball in $\mathbb{R}^{n}$; that is, $\operatorname{sop} \varphi=$ $B_{1}(0)$.

One expression of the form $\alpha=\left(\alpha_{1}, \ldots, \alpha_{n}\right)$ with $\alpha_{i}$ being a nonnegative integer, for each $i=1, \ldots, n$, is called a multiindex. For the multi-index $\alpha$ the order of $\alpha$ is defined, denoted by $|\alpha|$, by

$$
|\alpha|=\alpha_{1}+\cdots+\alpha_{n} .
$$

If $x=\left(x_{1}, \ldots, x_{n}\right) \in \mathbb{R}^{n}$ and $\alpha=\left(\alpha_{1}, \ldots, \alpha_{n}\right)$ are one multiindex, then it is defined that

$$
\partial^{\alpha} \mu:=\frac{\partial^{|\alpha|} \mu}{\partial x_{1}^{\alpha_{1}} \cdots \partial x_{n}^{\alpha_{n}}}, \quad x^{\alpha}=x_{1}^{\alpha_{1}} x_{2}^{\alpha_{2}} \cdots x_{n}^{\alpha_{n}} .
$$

Convergence in $\mathscr{D}(\Omega):\left(\alpha_{n}\right)_{n=1}^{\infty}$ being one succession of functions in $\mathscr{D}(\Omega), \varphi_{n} \rightarrow \varphi \in \mathscr{D}(\Omega)$, when $n \rightarrow \infty$ if

(a) there is one compact $K \subset \Omega$ so that for each $n \operatorname{sop} \varphi_{n} \subset$ $K$

(b) $\partial^{\alpha} \varphi_{n} \rightarrow \partial^{\alpha} \varphi$ uniformly in $K$, for each multi-index $\alpha$.

The uniform convergence in $K$ of the succession $\left(\partial^{\alpha} \varphi_{n}\right)_{n=1}^{\infty}$ means that

$$
\sup _{x \in K}\left|\partial^{\alpha} \varphi_{n}-\partial^{\alpha} \varphi(x)\right| \longrightarrow 0
$$

when $n \rightarrow \infty$. One application $f$ is continued in $\mathscr{D}(\Omega)$, meaning that each succession $\left(\varphi_{n}\right)_{1}^{\infty}$, with limit $\varphi$, has $\left\langle f, \varphi_{n}\right\rangle \rightarrow\langle f, \varphi\rangle$, when $n \rightarrow \infty$.

For each open set $\Omega$ in $\mathbb{R}^{n}$.

The application $T: D(\Omega) \rightarrow \mathbb{C}$ is one distribution if

(a) $T$ is linear;

(b) for each compact $K \subset \Omega$ there is a constant $C_{K}>0$ and one nonnegative integer $m$ (depends of $K$ ) as

$$
\left|\left\langle T, \varphi_{n}\right\rangle\right| \leq C_{K} \sum_{|\alpha| \leq m} \sup _{x \in K}\left|\partial^{\alpha} \varphi(x)\right|,
$$

for each $\varphi \in D_{K}(\Omega)$ and for each multi-index $\alpha$.

One distribution is a linear and continuous functional over $D(\Omega)$. The space for all distributions over $\Omega$ is denoted by $D^{\prime}(\Omega)$. This means, $D^{\prime}(\Omega)=\mathscr{L}(D(\Omega), \mathbb{C})$ is the dual of $D(\Omega)$.

2.2. Conditions. The distributions of compact support are defined as linear continuous functions over $C^{\infty} K$ with a defined topology over the space by the uniform convergence.

Then the function

$$
f|\varphi|=\int_{-\infty}^{\infty} f(x) \delta(x)
$$

which converges $\forall \delta(x) \in K$ is called a "regular distribution."
2.3. Dirac's Delta. The concept of the Dirac's delta "function," also called unitary impulse function, is useful to model situations like, for example, where there is a mechanical system over which an external large magnitude force acts during a brief moment of time. In an extreme case that this force be concentrated in one point, it would be possible to represent it by Dirac's delta. In all cases when a very intense external signal is applied, during a very short time interval, the use of the impulsive signal $\delta(x)[8]$ is introduced to model these situations.

Dirac's delta can be expressed symbolically:

$$
\delta(x)= \begin{cases}0 ; & x \neq 0 \\ \infty ; & x=0\end{cases}
$$

so that the integral of $\delta(x)$ is normalized to the unity; this means

$$
\int_{-\infty}^{\infty} \delta(x) d x=1
$$

The $\delta$ function satisfies the property

$$
\int_{-\infty}^{\infty} f(x) \delta(x) d x=f(0)
$$

for all continuous function $f(x)$ :

$$
\begin{aligned}
\int_{-\infty}^{\infty} f(x) \delta(x) d x & =\int_{-\varepsilon}^{\varepsilon} f(x) \delta(x) d x \\
& \approx f(0) \int_{-\varepsilon}^{\varepsilon} \delta(x) d x .
\end{aligned}
$$

The approximation has a better behavior as $\varepsilon \rightarrow 0$; however

$$
\int_{-\varepsilon}^{\varepsilon} \delta(x) d x=1
$$

for all the values of $\varepsilon$ where $\delta(x)=0 \forall x \neq 0, \delta(x)$ is normalized. Then when solving the limit when $\varepsilon \rightarrow 0$ is obtained,

$$
\int_{-\varepsilon}^{\varepsilon} f(x) \delta(x) d x=f(0) .
$$

This integral is called the filter property from the delta function, when $\delta(x)$ is acting as a filter, selecting from all the possible values of $f(x)$ the value in the point $x=0$.

In [10], the integral is evaluated using the following argument: due to $\delta(x)=0$ for all $x \neq 0$, we can change the integration limits in the following $(-\varepsilon)$ and $(\varepsilon)$, where $\varepsilon$ is a positive infinitesimal number. Even more, assuming that $f$ is continuous in $x=0$, its values inside the selected interval $(-\varepsilon, \varepsilon)$ are approximated to $f(0)$, and we can make the following approximation:

$$
\begin{aligned}
\int_{-\infty}^{\infty} f(x) \delta(x) d x & =\lim _{\varepsilon \rightarrow 0} \int_{-\varepsilon}^{\varepsilon} f(x) \delta(x) d x \\
& =f(0) \int_{-\varepsilon}^{\varepsilon} \delta(x) d x \\
\int_{-\infty}^{\infty} \delta(x) d x & =1,
\end{aligned}
$$


and, changing limits,

$$
\begin{aligned}
& \lim _{\varepsilon \rightarrow 0} \frac{1}{\varepsilon} \int_{-\varepsilon / 2}^{\varepsilon / 2} f(x) d x=\left.\lim _{\varepsilon \rightarrow 0} \frac{1}{\varepsilon} F(x)\right|_{+\varepsilon / 2} ^{-\varepsilon / 2} \\
& =\lim _{\varepsilon \rightarrow 0} \delta \frac{f(\varepsilon / 2)-F(-\varepsilon / 2)}{\varepsilon}=F^{\prime}(0)=f(0) .
\end{aligned}
$$

Then

$$
\int_{-\infty}^{\infty} f(x) \delta(x) d x=f(0) .
$$

\section{Convergency Analysis}

The convergence of the estimator will be studied using the distributions theory, the Lebesgue convergence theorem, and the convolution properties. We will study the estimator convergence using the distribution theory, Lebesgue convergence theorem, and the convolution properties.

3.1. Delta Succession. For the solution of the problem associated with the use of (12), an alternative function must be sought, which is to define the function $\delta(x)$ as the function that satisfies the filter propriety

$$
\int_{-\infty}^{\infty} f(x) \delta(x) d x=f(0)
$$

for all continuous functions $f(x)$, symbolically written

$$
f(x) \delta(x)=f(0) .
$$

One type of delta function is

$$
\begin{aligned}
\delta_{n}(x) & =\frac{n}{\pi} \frac{1}{1+\pi^{2} n^{2}} ; \\
\lim _{n \rightarrow \infty} \delta_{n}(x) & \longrightarrow \delta(x),
\end{aligned}
$$

which satisfies the equation

$$
\lim _{n \rightarrow \infty} \int_{-\infty}^{\infty} f(x) \delta_{n}(x) d x=f(0) .
$$

3.2. Lebesgue Convergence Theorem. Let $\left\{f_{n}\right\}$ be one function succession, $f_{n} \in L^{1}(T)$, such that $f_{n}(x) \rightarrow f(x)$ for some measurable $f$. If there exists $p \in L^{1}(T)$, such that $\left|f_{n}(x)\right| \leq$ $p(x)$, for all $p$, then

$$
\begin{aligned}
f & \in L^{1}(T), \\
\lim _{n \rightarrow \infty}\left\|f_{n}-f\right\|_{1} & =0, \\
\int_{T} f(x) d x & =\lim _{n \rightarrow \infty} \int_{T} f_{n}(x) d x .
\end{aligned}
$$

Applying the convergence theorem, according to [11], $f_{n}$ is a succession of Lebesgue integrable functions in an $I$ interval. Assuming that $f_{n}$ converges in $I$, to some limit function, and there is one nonnegative Lebesgue $p$ function in $I$ so that, for all $n \geq 1$,

$$
\left|f_{n}(x)\right| \leq p(x),
$$

then the limit function $f$ belongs to a Lebesgue function; the succession $\left|f_{n}\right|$ converges

$$
\int_{I} f=\lim _{n \rightarrow \infty} \int_{I} f_{n} .
$$

Consequently [12],

$$
\lim _{n \rightarrow \infty}\left\{\delta_{n}(t)\right\} \longrightarrow \delta(t),
$$

applying the convolution, which is always a function, that switches with the translations and derivations, also with the regularized effect. Let $\mu$ be one distribution in $\mathbb{R}^{n}$, and $\phi, \psi \in$ $D\left(\mathbb{R}^{n}\right)$; then

$$
\begin{gathered}
\tau_{x}(\mu * \phi)=\left(\tau_{x} \mu\right) * \phi=\mu *\left(\tau_{x} \phi\right) \in\left(\mathbb{R}^{n}\right) \\
\mu * \phi \in C^{\infty}\left(\mathbb{R}^{n}\right), \\
D^{\alpha}(\mu * \phi) \\
=\left(D^{\alpha} \mu\right) * \phi \\
=\mu *\left(D^{\alpha} \phi\right) .
\end{gathered}
$$

For all multi-index $\alpha$,

$$
\begin{aligned}
\mu *(\phi * \psi) & =(\mu * \phi) * \psi \\
\delta * \phi & =\phi,
\end{aligned}
$$

where $\delta$ is Dirac's delta [13].

According to Hernandez (1994), let $\mu \in D^{\prime}\left(\mathbb{R}^{n}\right), v \in$ $D^{\prime}\left(\mathbb{R}^{n}\right)$, and $w \in D^{\prime}\left(\mathbb{R}^{n}\right)$.

If at least one of the distributions $\mu$ and $v$ has compact support, then

(i) $\mu * v=v * \mu$

(ii) $\operatorname{sop}(\mu * s v) \subset \operatorname{sop}(\mu)+\operatorname{sop}(v)$.

Let $\alpha$ be one multi-index; then,

$$
\begin{aligned}
\text { (i) } D^{\alpha} v & =\left(D^{\alpha} \delta\right) * \mu \\
\text { (ii) } \delta * v & =v .
\end{aligned}
$$

If $\delta$ has compact support

$$
(\operatorname{sop}(\delta)=\{0\}),
$$

then,

$$
\begin{aligned}
D^{\alpha}(\mu) * \phi & =\mu *\left(D^{\alpha} \phi\right)=\mu * D^{\alpha}(\delta * \phi) \\
& =\mu * D^{\alpha}(\delta) * \phi=\mu * D^{\alpha} \delta * \phi \\
& =D^{\alpha} \delta * \mu * \phi .
\end{aligned}
$$


Then,

$$
D^{\alpha} \mu=\left(D^{\alpha} \delta\right) * \mu .
$$

Assuming $|\alpha|=0$, then

$$
\begin{aligned}
& D^{\alpha} \mu=\mu, \\
& D^{\alpha} \delta=\delta
\end{aligned}
$$

gives

$$
\mu * \delta=\mu .
$$

Thus, it can be observed that $\delta$ is the unit element for the convolution and is considered as an algebraic operation.

Now we will apply the distributions theory to the convergence study of the estimation technique proposed in Moreno et al. (2010).

In that case the transition matrix, assuming an LTI system, is

$$
P(t) \triangleq \int_{t_{0}}^{t} e^{A^{T}(\tau-t)} C^{T} C e^{A^{(\tau-t)}} \delta\left(\tau-t_{i}\right) d \tau
$$

and the Lebesgue convergence theorem gives

$$
\lim _{n \rightarrow \infty} \int_{t_{0}}^{t} e^{A^{T}\left(\tau-t_{0}\right)} C C^{T} e^{A^{\left(\tau-t_{0}\right)}} \delta_{n}\left(\tau-t_{i}\right) d \tau .
$$

Applying the convolution we obtain

$$
\lim _{n \rightarrow \infty} \int_{t_{0}}^{t} \delta_{n}\left(\tau-t_{i}\right) e^{A^{T}\left(\tau-t_{i}\right)} C C^{T} e^{A^{\left(\tau-t_{0}\right)}} d \tau,
$$

and solving the limit

$$
\int_{t_{0}}^{t} \delta\left(\tau-t_{i}\right) e^{A^{T}\left(\tau-t_{0}\right)} C C^{T} e^{A^{\left(\tau-t_{0}\right)}} d \tau,
$$

obtaining

$$
\int_{t_{0}}^{t} e^{A^{T}(\tau-t)} C C^{T} e^{A^{(\tau-t)}} d \tau,
$$

where $P(t)$ meets the Lyapunov stability condition, meaning

$$
\left\|P\left(t_{i}\right)-P\left(t_{j}\right)\right\| \leq L\left\|t_{i}-t_{j}\right\|
$$

for all LTI in $[0, T]$; therefore the system is bounded.

$P(t)$ has a monotonously exponential behavior and converges consequently to the model of the plant (3).

From the following expressions:

$$
\begin{aligned}
\dot{x}(t) & =A x(t)+B u(t), \\
\dot{\hat{x}}(t) & =A \hat{x}(t)+B u(t)+S(t) C^{T}[Y(t)-C \hat{x}(t)], \\
e & =x(t)-\widehat{X}(t), \\
\dot{e}(t) & =A e(t)-S(t) C^{T}[y(t)-C \hat{x}(t)], \\
\dot{e}(t) & =A e(t)-S(t) C^{T} C e(t), \\
\dot{e}(t) & =\left(A-S(t) C^{T} C\right) e(t),
\end{aligned}
$$

where

$$
C^{T} C=\lambda ; \quad \lambda \in \mathbb{R}
$$

obtaining,

$$
\dot{e}(t)=(A-S(t)) e(t),
$$

according to [14], the matrix $A$ is a constant and Hurwitz and the matrix $S(t)$ is time-varying such that

$$
\begin{aligned}
& S(t) \longrightarrow 0 ; \quad t \longrightarrow \infty \\
& \int_{0}^{\infty}\|S(t)\| d t<\infty .
\end{aligned}
$$

$S(t)$ is a matrix that is set in each iteration, reaching in some $t_{i}$, but does not require further adjustment.

Then system (46) is globally exponentially stable [14].

\section{Conclusions}

We started from state estimators based model for LTI systems with event detection to which convergence was determined.

$P(t)$ converges to the family of functions given in (42), so the estimated $x(t)$ converges to the $x(t)$ plant model for $t(0, T)$.

In future studies, assuming that the adjustments made to $S(t)$ are becoming smaller, it would be possible to determine the least $(j)$, such that $S\left(t_{i}\right)=S\left(t_{j+i}\right)$.

The distributions theory was used; this generalizes the function notion, extending the concept of derivative to all locally integrable functions; permitting the topology change (homeomorphism), with the purpose of giving it a mathematic rigorousness.

The model convergence of estate estimators was obtained after applying this theory, allowing future applications using this type of state estimators.

This study offers great advantages to systems where the information is limited, where continuous sensors are expensive or less reliable, replacing them by binary detectors with more resistant characteristic and less expenses.

\section{Competing Interests}

The authors declare that they have no competing interests.

\section{Acknowledgments}

Gratitude is presented to Dr. Jairo Alberto Villegas G, for his valued contribution in the convergence study realized in this paper.

\section{References}

[1] L. Y. Wang, G. Xu, and G. Yin, "State reconstruction for linear time-invariant systems with binary-valued output observations," Systems and Control Letters, vol. 57, no. 11, pp. 958-963, 2008 . 
[2] L. Y. Wang, C. Li, G. G. Yin, L. Guo, and C.-Z. Xu, "State observability and observers of linear-time-invariant systems under irregular sampling and sensor limitations," IEEE Transactions on Automatic Control, vol. 56, no. 11, pp. 2639-2654, 2011.

[3] K. Astrom, Directions in Mathematical Systems Theory and Optimization, Springer, Berlin, Germany, 2003.

[4] J. A. Moreno, M. J. Betancur, and M. A. Tamayo, "Observation of linear time-invariant systems with lebesgue sampling," in Proceedings of the IEEE ANDESCON, pp. 1-6, IEEE, Bogotá, Colombia, September 2010.

[5] M. Biqiang and G. Jin, "Identification of linear continuoustime systems under irregular and random output sampling," Automatica, vol. 60, pp. 100-114, 2015.

[6] J. Guo and Y. Zhao, "Recursive projection algorithm on FIR system identification with binary-valued observations," Automatica, vol. 49, no. 11, pp. 3396-3401, 2013.

[7] Y. Yajing, D. Hongli, Z. Wangb, W. Rena, and F. E. Alsaadic, "Design of non-fragile state estimators for discrete time-delayed neural networks with parameter uncertainties," Neurocomputing, vol. 182, pp. 18-24, 2016.

[8] R. Strichartz, A Guide to Distribution Theory and Fourier Transforms, vol. 1, World Scientific, River Edge, NJ, USA, 1994.

[9] F. Hernandez, Teoría de distribuciones [M.S. thesis], Universidad Nacional de Colombia, Bogotá, Colombia, 1994.

[10] R. Romero, "Funciones especiales y transformadas integrales," Tech. Rep., Universidad Autonónoma Metropolitana Iztapalapa, Mexico City, Mexico, 2010.

[11] E. Agora, Integrales singulares en el análisis armónico [Máster thesis], Universitat de Barcelona, Barcelona, Spain, 2007.

[12] A. H. Zemanian, Distribution Theory and Trasform Analysis, vol. 1, Dover, New York, NY, USA, 1987.

[13] G. Lopez, Apuntes de Teoría de Distribuciones, vol. 1, Universidad de Granada, Granada, Spain, 2008.

[14] J. J. E. W. L. Slotine, Applied Nonlinear Control, edited by: P. Hall, Pearson Education, Upper Saddle River, NJ, USA, 1st edition, 1991. 


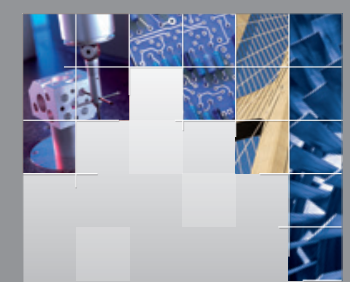

\section{Enfincering}
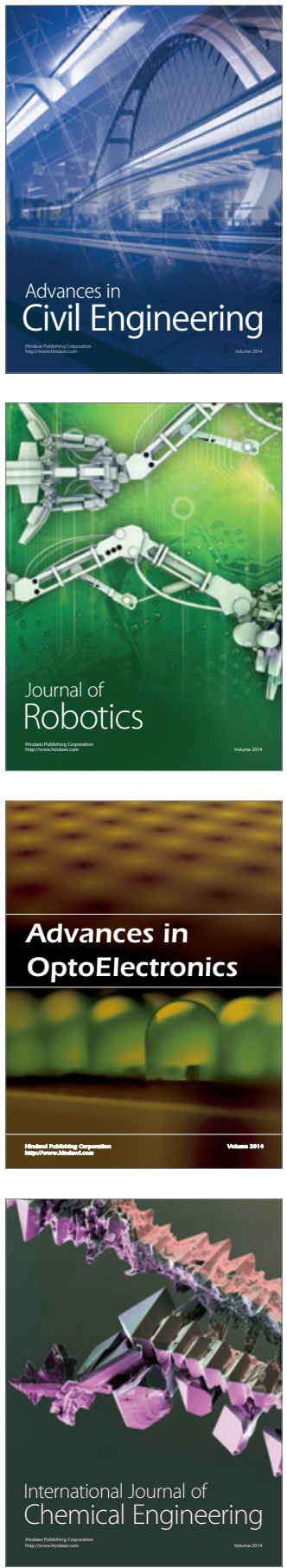

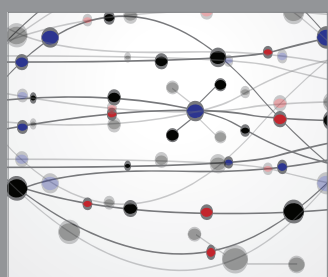

The Scientific World Journal

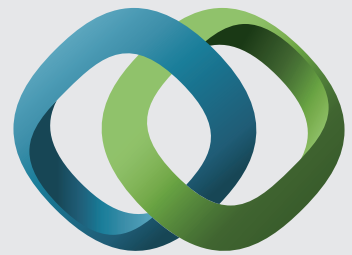

\section{Hindawi}

Submit your manuscripts at

http://www.hindawi.com
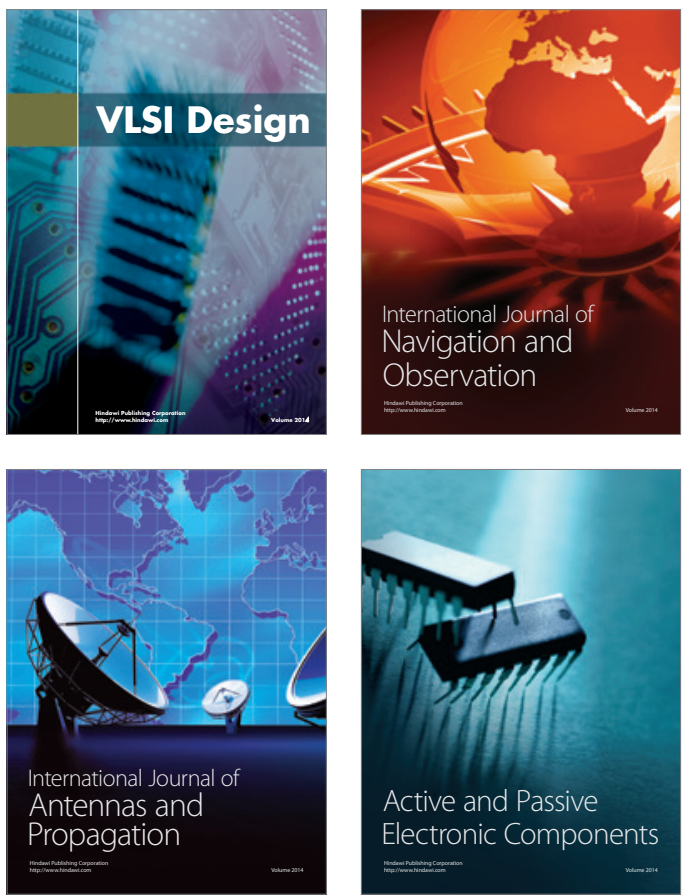
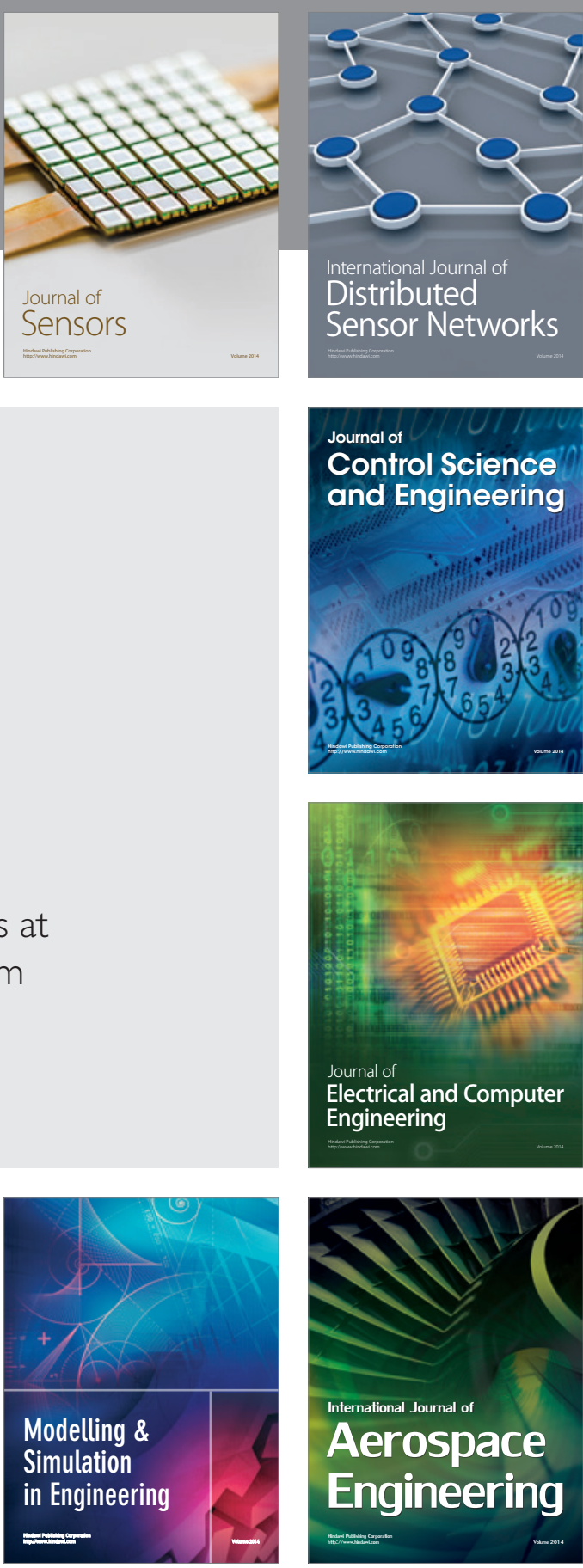

International Journal of

Distributed

Sensor Networks

Journal of

Control Science

and Engineering
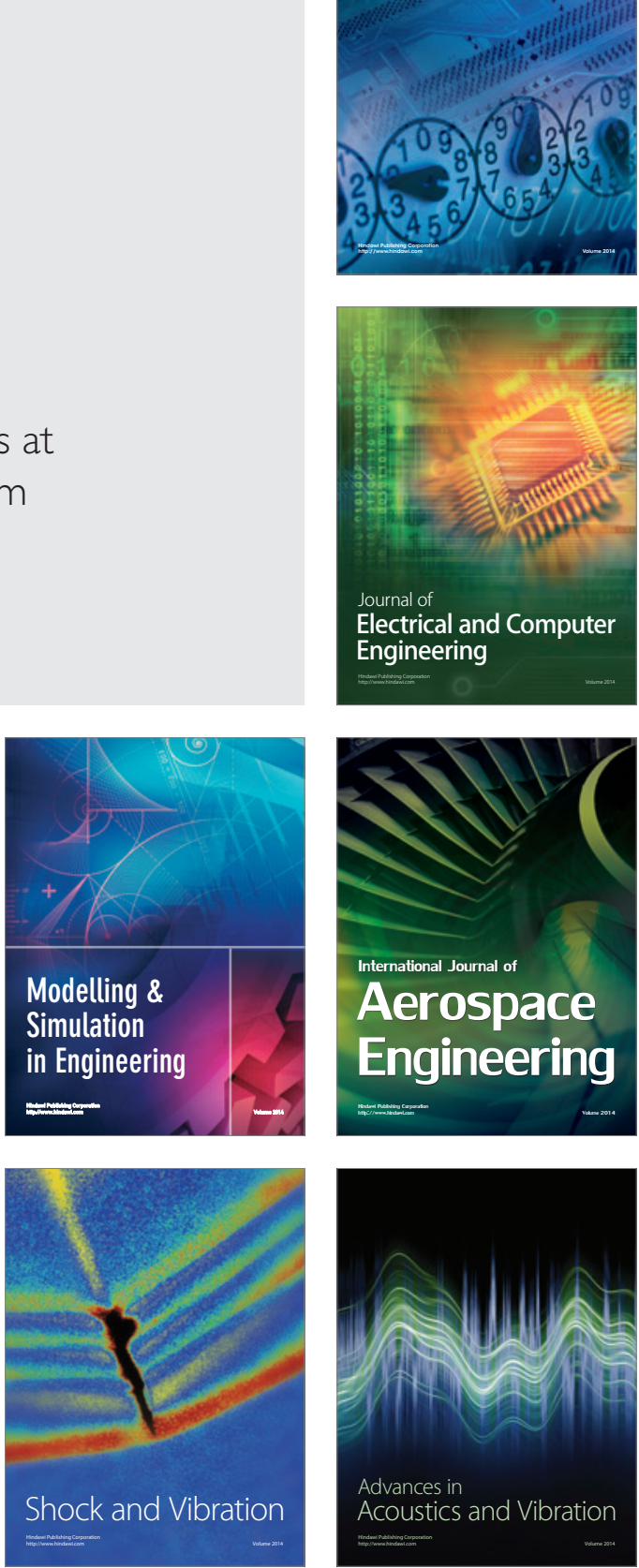